\title{
Tracking the mutant: forecasting and nowcasting COVID-19 in the UK in 2021
}

\author{
Andrew Harvey ${ }^{\dagger}$, Paul Kattuman* \& Craig Thamotheram ${ }^{\ddagger}$ \\ ${ }^{\dagger}$ Faculty of Economics, University of Cambridge \\ ${ }^{\dagger}$ Cambridge Judge Business School, University of Cambridge \\ ${ }^{\ddagger}$ National Institute of Economic and Social Research
}

\begin{abstract}
A new class of time series models is used to track the progress of the COVID-19 epidemic in the UK in early 2021. Models are fitted to England and the regions, as well as to the UK as a whole. The growth rate of the daily number of cases and the instantaneous reproduction number are computed regularly and compared with those produced by SAGE. The results from figures published each day are compared with results based on figures by specimen date, which may be more accurate but are subject to substantial revisions. It is then shown how data from the two different sources can be combined in bivariate models.
\end{abstract}

Keywords: Data revisions; epidemic; Kalman filter; reproduction number (R); state space model; stochastic trend.

JEL codes: C22, C32.

Corresponding author: Paul Kattuman. Email: p.kattuman@jbs.cam.ac.uk 


\section{Introduction}

The application of classical time series methods to data on epidemics is relatively undeveloped. Most of the emphasis has been on building models to simulate the path of an epidemic under different assumptions about behaviour and policies and the forecasting performance has often been unimpressive; see Avery et al (2020) and Ioannides et al (2020). Here we show how a new class of time series models can be used to track the progress of an epidemic and forecast key indicators. The methods draw much of their inspiration from econometrics, but take into account the special characteristics of time series for epidemics.

The univariate time series model described in Harvey and Kattuman (2020a) - hereafter HK - fits a trend to the logarithm of the growth rate of the cumulated series of the target variable, which is usually new cases, hospital admissions or deaths. Allowing this trend to be time-varying introduces flexibility which, in the context of an epidemic, enables the effects of changes in policy and population behaviour to be tracked. Such stochastic trend models are a standard econometric tool and they are easily handled within a state space framework. Application of the Kalman filter enables nowcasts and forecasts of variables of interest, such as the growth rate of the daily number of cases and the instantaneous reproduction number, to be made. Estimation of the models is by maximum likelihood and goodness of fit can be assessed by standard statistical test procedures.

This article describes our experience tracking the progress of the COVID-19 epidemic in the UK in early 2021. This period is of considerable interest because a new variant of the virus appeared in the south-east of England in December 2020 and started to spread throughout the country. The lockdown of January 5th 2021 was partially in response to this new variant. The number of new cases quickly rose to a peak around the beginning of the new year and then started to fall. The ability of models to respond to these movements in a timely fashion is clearly important. Here we investigate how our models fared by showing how the response, as captured by both nowcasts and forecasts, adapted to observations available on a daily basis. ${ }^{1}$

We first examined the results for the country as a whole before moving on to monitor the regions. Regional variation is significant because in October some areas of the country, such as north-west England, were particularly hard hit whereas the big rises in December came primarily from the new variant and were mainly in the south-east. There are systematic movements in daily observations according to the day of the week with the figures for the weekend tending to be lower. Our model is able to take account of these movements without

\footnotetext{
${ }^{1}$ This methodology forms the basis for the weekly projections of new cases and the $\mathrm{R}$ number for the United Kingdom, its constituent nations and the regions of England, published weekly by NIESR from 18 February 2021 (https://www.niesr.ac.uk/latest-weekly-covid-19-tracker).
} 
using seven-day moving averages which tend to result in a delayed response when there are rapid upward or downward movements.

Multivariate state space models can combine information in different series. There are two data sources for new COVID cases. One is the figure published each day, while the other is by specimen date. The second series is subject to substantial revisions as new data are processed and the series only settles down after about three days. However, it may be a better indicator of the spread of the epidemic and so the question arises as to whether the information it contains can be combined with that in the published data. This is essentially a question of combining different 'vintages', something which is often done with economic data. Sometimes the observations are made in a different way and at different frequencies, for example by surveys; see Harvey and Chung (2000) and, more recently, Anesti et al (2018). Our treatment of published and specimen data owes much to this literature but there are some novel features, primarily concerned with time-varying slopes and the notion of balanced growth. The methods may be generalized to deal with leading indicators as in Harvey (2020).

Section 2 of the paper reviews the model and explains how estimates of the growth rate of daily numbers can be made and how these yield corresponding estimates of instantaneous reproduction number, $R_{t}$. Our experience with UK data in January is reported in Section 3 and the multivariate models are described and implemented in Section 4.

\section{Forecasting and nowcasting with the dynamic Gom- pertz model}

The observational model uses data on the time series of the cumulated total of confirmed cases or deaths, $Y_{t}, t=0,1, \ldots, T$, and the daily change. HK show how the theory of generalized logistic growth curves suggests models for $\ln y_{t}$, where $y_{t}=\Delta Y_{t}=Y_{t}-Y_{t-1}$, and the logarithm of the growth rate of the cumulated series, $\ln g_{t}$, where $g_{t}=y_{t} / Y_{t-1}$ or $\Delta \ln Y_{t}$. For the special case of the Gompertz growth curve, the implication is that $\ln g_{t}$ follows a downward linear trend. However, additional flexibility is needed to cope with situations where there are recurrent waves. This may be achieved by a stochastic, or time-varying, trend so that

$$
\ln g_{t}=\delta_{t}+\varepsilon_{t}, \quad \varepsilon_{t} \sim N I D\left(0, \sigma_{\varepsilon}^{2}\right), \quad t=1,2, \ldots, T
$$

where $^{2}$

\footnotetext{
${ }^{2} \mathrm{HK}$ had a negative sign in front of $\gamma$ in (1) and (2) because in a growth curve the growth rate is always falling so it is more convenient to let $\gamma$ be positive. This ceases to be the case once there are second waves.
} 


$$
\begin{aligned}
\delta_{t}=\delta_{t-1}+\gamma_{t-1}+\eta_{t}, & & \eta_{t} \sim N I D\left(0, \sigma_{\eta}^{2}\right), \\
\gamma_{t}=\gamma_{t-1}+\zeta_{t}, & & \zeta_{t} \sim N I D\left(0, \sigma_{\zeta}^{2}\right),
\end{aligned}
$$

and the normally distributed irregular, level and slope disturbances, $\varepsilon_{t}, \eta_{t}$ and $\zeta_{t}$, respectively, are mutually independent. When $\sigma_{\zeta}^{2}$ is positive but $\sigma_{\eta}^{2}=0$ the trend is an integrated random walk (IRW). HK found IRW trend to be particularly useful for tracking an epidemic and it will be adopted in the applications here. The speed with which a trend adapts to a change depends on the signal-noise ratio, which for the IRW is $q_{\zeta}=\sigma_{\zeta}^{2} / \sigma_{\varepsilon}^{2}$; the trend is deterministic when $q=0$.

Allowing $\gamma_{t}$ to change over time means that the progress of the epidemic is no longer tied to the proportion of the population infected as it would be if $Y_{t}$ followed a deterministic growth curve. Instead the model adapts to movements brought about by changes in behaviour and policies. If $\gamma_{t}$ falls to zero, the growth in $Y_{t}$ becomes exponential whereas a positive $\gamma_{t}$ means that the growth rate is increasing.

Stochastic trend models can be estimated using techniques based on state space models and the Kalman filter (KF); see Durbin and Koopman (2012) and Harvey (1989). The computations for the multivariate model were performed using the STAMP package of Koopman et al. (2020) while the results reported in Section 3 were obtained with a new program in the $\mathrm{R}$ language specifically written for this project. The KF outputs the estimates of the state vector $\left(\delta_{t}, \gamma_{t}\right)^{\prime}$. Estimates of the state at time $t$ conditional on information up to and including time $t$ are denoted $\left(\delta_{t \mid t}, \gamma_{t i t}\right)^{\prime}$ and given by the contemporaneous filter while the predictive filter outputs $\left(\delta_{t+1, t}, \gamma_{t+1, t}\right)^{\prime}$. The smoother estimates the state at time $t$ based on all $T$ observations in the series and is denoted $\left(\delta_{t \mid T}, \gamma_{t \mid T}\right)^{\prime}$. Estimation of the unknown variance parameters is by maximum likelihood. Tests for normality and residual serial correlation are based on the one-step ahead prediction errors, $v_{t}=\ln g_{t}-\delta_{t \mathrm{t}-1}, t=3, \ldots, T$.

Additional components, such as day of the week effects, can be added to (1). These may be deterministic or stochastic. Stationary autoregressive or ARMA components may also be included as may explanatory variables, including interventions. However, isolated outliers are most easily handled by treating them as missing observations.

Remark 1 When the observations on daily cases or deaths are small, a negative binomial distribution for $y_{t}$, conditional on past observations including $Y_{t-1}$, may be appropriate. HK show how the model may be modified to deal with this possibility for a univariate time series. Software can be found in Lit et al (2020). Estimates of the state based on small numbers are likely to be unreliable but if the KF is to operate during periods when numbers are small, as they were for COVID-19 cases in the summer of 2020, it may be better to set $v_{t}=$ 
$g_{t} \exp \left(-\delta_{t, t-1}\right)-1$ rather than to treat the observation as missing.

\subsection{Forecasting and nowcasting the growth rate of daily observations and $R$}

The direction in which an epidemic is moving is best tracked by nowcasts and forecasts of $g_{y, t}$, the growth rate of $y_{t}$. Harvey and Kattuman (2020b) construct the nowcast of $g_{y, t}$ from the filtered estimates in the state space model, (1) and (2). Thus $g_{y, t \mid t}=g_{t \mid t}+\gamma_{t \mid t}$. These estimates can be translated into estimates of the instantaneous reproduction number $R_{t}$, in a number of ways, as described in Wallinga and Lipsitch (2007). Harvey and Kattuman (2020b) argue that the most useful for COVID-19 are

$$
\widetilde{R}_{t, \tau}=1+\tau g_{y, t \mid t} \quad \text { and } \quad \widetilde{R}_{\tau, t}^{e}=\exp \left(\tau g_{y, t \mid t}\right),
$$

where $\tau$ is the generation interval, that is the number of days that must elapse before an infected person can transmit the disease. The nowcasts of $y_{t}$ peak when $g_{y, t \mid t}=0$, corresponding to $\widetilde{R}_{t, \tau}=\widetilde{R}_{\tau, t}^{e}=1$.

For tracking and forecasting the epidemic all that is needed are estimates of $g_{y, t}$. The estimates of $R_{t}$ are a by-product. Despite being dependent on assumptions about the generation interval, estimates of $R_{t}$ have become the main metric for reporting the state of the epidemic.

Predictions of $g_{y, t}$, and hence of $R_{t}$, are given by

$$
g_{y, T+\ell \mid T}=\exp \delta_{T+\ell \mid T}+\gamma_{T+\ell \mid T}=\exp \left(\delta_{T \mid T}+\gamma_{T \mid T} \ell\right)+\gamma_{T \mid T}, \quad \ell=1,2, .
$$

If $\gamma_{T \mid T}$ is zero, the growth of $y_{t}$ is exponential and it is helpful to characterize it by the doubling time, $\ln 2 / g_{y, T \mid T}=0.693 \exp \left(-\delta_{T \mid T}\right)$.

When $\exp \delta_{T \mid T}+\gamma_{T \mid T}>0$, so that the nowcast $\widetilde{g}_{y, T \mid T}$ is positive and the estimates of $R_{t}$ given by (3) are greater than one, there is still a saturation level so long as $\gamma_{T \mid T}$ is negative;

correspondingly, as $T \rightarrow \infty, \widetilde{R}_{\tau, T+\ell \mid T}^{e} \rightarrow \exp \left(\tau \gamma_{T \mid T}\right)<1$. Hence a negative $\gamma_{T \mid T}$ signals a flattening of the curve and an upcoming peak in $y_{t}$.

Remark 2 The basic forecasts are made with the estimates of $\delta_{T}$ and $\gamma_{T}$. However, alternative scenarios in which $\gamma_{t}$ is assumed to evolve in a certain way, perhaps to reflect changing behaviour and policies, may also be envisaged. If a future scenario arises in terms of a time path for $R_{T+\ell \mid T}$, it can easily be translated into one for $\gamma_{T+\ell \mid T}$. The time path for $\gamma_{T+\ell \mid T}$ leads directly to the forecasting equations of (10) and so no simulations are needed for the predictions of $y_{T+\ell}$. 


\subsection{Sampling variability of nowcasts and forecasts}

Harvey and Kattuman (2020b) show that the conditional distribution of nowcasts of $g_{y, t}$ can be approximated by the conditional distribution of $\gamma_{t}$, which is normal with mean $\gamma_{t \mid t}$ and variance $\sigma_{\gamma, t \mid t}^{2}$, both of which are produced by the $\mathrm{KF}$.

When $\widetilde{R}_{t, \tau}$ is defined as $1+\tau g_{y, t}$, its distribution, conditional on current and past observations, can be treated as $N\left(g_{y, t \mid t}, \tau^{2} \sigma_{\gamma, t \mid t}^{2}\right)$. On the other hand the conditional distribution of $\widetilde{R}_{\tau, t}^{e}$ is lognormal with mean

$$
E_{t}\left(\widetilde{R}_{\tau, t}^{e}\right)=\exp \left(\tau\left(g_{t \mid t}+\gamma_{t \mid t}+(\tau / 2) \sigma_{\gamma, t \mid t}^{2}\right)\right)
$$

and standard deviation

$$
S D_{t}\left(\widetilde{R}_{\tau, t}^{e}\right)=E_{t}\left(\widetilde{R}_{\tau, t}^{e}\right) \sqrt{\left(\exp \tau^{2} \sigma_{\gamma, t \mid t}^{2}-1\right)}
$$

Note that $\exp \tau^{2} \sigma_{\gamma, t \mid t}^{2}-1 \simeq \tau^{2} \sigma_{\gamma, t \mid t}^{2}$ so when $E_{t}\left(\widetilde{R}_{t, \tau}\right)$ is close to one, $S D_{t}\left(\widetilde{R}_{\tau, t}^{e}\right) \simeq S D_{t}\left(\widetilde{R}_{t, \tau}\right)$. The probability that $R_{t}$ exceeds one is $\operatorname{Pr}\left(g_{y, t \mid t}>0\right)$ and this does not depend on $\tau$ or the formula used to estimate $R_{t}$ from $g_{y, t \mid t}$.

Remark 3 For the Spanish flu data Chowell et al (2007) discuss two approaches to estimating $R_{t}$ based on SEIR models, the more complex one having eight nonlinear differential equations. They also use the Bayesian method of Bettencourt and Ribeiro (2008). Estimates of $R_{t}$ obtained from the model discussed at the end of this section are not out of line with those reported by Chowell et all (2007) and they are simpler, more transparent and open to diagnostic checks on the statistical assumptions.

As with nowcasts, the predictive distribution of $g_{y, T+\ell}$, and hence of $R_{T+\ell}$, can be approximated from the conditional distribution of $\gamma_{T+\ell}$ given observations up to and including time $T$. This is Gaussian with mean $\gamma_{T \mid T}$ and variance $\sigma_{\gamma, T+\ell \mid T}^{2}$. These estimates are produced by the predictive equations of the KF as in Harvey (1989, p 147, eq 3.5.5). For an IRW trend it can be shown that

$$
\operatorname{Var}_{T}\left(g_{y, T+\ell}\right) \simeq \operatorname{Var}_{T}\left(\gamma_{T+\ell}\right)=\operatorname{Var}_{T}\left(\gamma_{T}\right)+\ell \sigma_{\zeta}^{2}=\sigma_{\gamma, T \mid T}^{2}+\ell q \sigma_{\varepsilon}^{2}
$$

when the effect of the daily component is not included. The factor by which the variance of an $\ell$ step ahead forecast of $R_{t}=1+\tau g_{y, t}$ is inflated above that of the variance of the corresponding nowcast is the same as it is for $g_{y, t}$. For example, when $q=0.005, \sigma_{\gamma, T \mid T}^{2}=$ 0.001 and $\sigma_{\varepsilon}^{2}=0.02$, expression (7) indicates that the SDs of $g_{y t}$ and $R_{t}$ will increase by $30 \%$ for $\ell=7$ and $55 \%$ for $\ell=14$. 
The probability that $R_{T+\ell}>1$ is $\operatorname{Pr}\left(g_{y, T+\ell}>0\right) \simeq \operatorname{Pr}\left(z>-g_{y, T+\ell \mid T} / S D_{y, T+\ell \mid T}\right)$ where $z \sim N(0,1)$ and $S D_{y, T+\ell \mid T}$ is the square root of $(7)$. Thus for new cases in England by date of publication, the nowcast made on January 18th was -0.048 , while the 14 day forecast was -0.054 . The value of $\sigma_{\gamma, T \mid T}^{2}$ for $q=0.005$, and a daily effect included, was 0.0004 , while $\sigma_{\varepsilon}^{2}$ was estimated to be 0.014 . These values give $\operatorname{Pr}\left(R_{T}>1\right) \simeq \operatorname{Pr}(z>0.048 / \sqrt{0.0004})=$ $\operatorname{Pr}(z>2.40)=0.008$ and $\operatorname{Pr}\left(R_{T+14}>1\right) \simeq \operatorname{Pr}(z>1.30)=0.10$.

The ability to make predictions offers a way to deal with reporting delay, as described in Abbott et al (2020, p3-4). If the observation for time $t-k$ is not available until time $t$, the current $R_{t}$ is better estimated by a $k$-step ahead forecast. Taking the parameter values of the previous paragraph gives an increase in the SD of $14 \%$ for $k=3$.

\subsection{Moving averages}

In the UK the current level of new infections or deaths is usually reported together with the seven day moving average (MA7) which is more stable than the daily figure and irons out the daily effects. The moving average figure is often divided by 100,000 so as to give a standardized measure. Estimates of $g_{y, t}$ and $R_{t}$ can be calculated directly from the moving average. For example

$$
\widehat{R}_{t, k, \tau}=\frac{\sum_{j=0}^{k-1} y_{t-j}}{\sum_{j=r}^{k+r-1} y_{t-j}}=\frac{\sum_{j=0}^{k-1} y_{t-j}}{\sum_{j=0}^{k-1} y_{t-r-j}}=1+r \widehat{g}_{y, t},
$$

where the sum in the denominator starts at a lag of four and the sums in the numerator and denominator may overlap. The lag of $r$ reflects the generation interval, which is number of days that elapse before an infected person can transmit the disease. The Robert Koch Institute estimator ${ }^{3}$ has $r=4$, and $k=4$ or 7 ; setting $k=7$ has the advantage of smoothing out the daily effect.

Following on from (8) estimates of $g_{y, t}$ can be calculated directly from the moving average. However, because the observations are best captured by a location/scale model in which the level is proportional to scale, estimates formed from the level have poor statistical properties. A better approach would be to take logarithms before averaging. Harvey and Kattuman (2020b, section 3.3) show that doing so would give a result much closer to that obtained from the model.

A disadvantage of using simple moving averages to track the epidemic is that they give the last seven observations equal weights and so can be slow to respond to upward or downward movements. By contrast the model deals directly with day of the week effects and so is able

\footnotetext{
${ }^{3}$ There is some prior nowcasting to to account for reporting delays; the methodology is based on Höhle and an der Heiden ( 2014)
} 


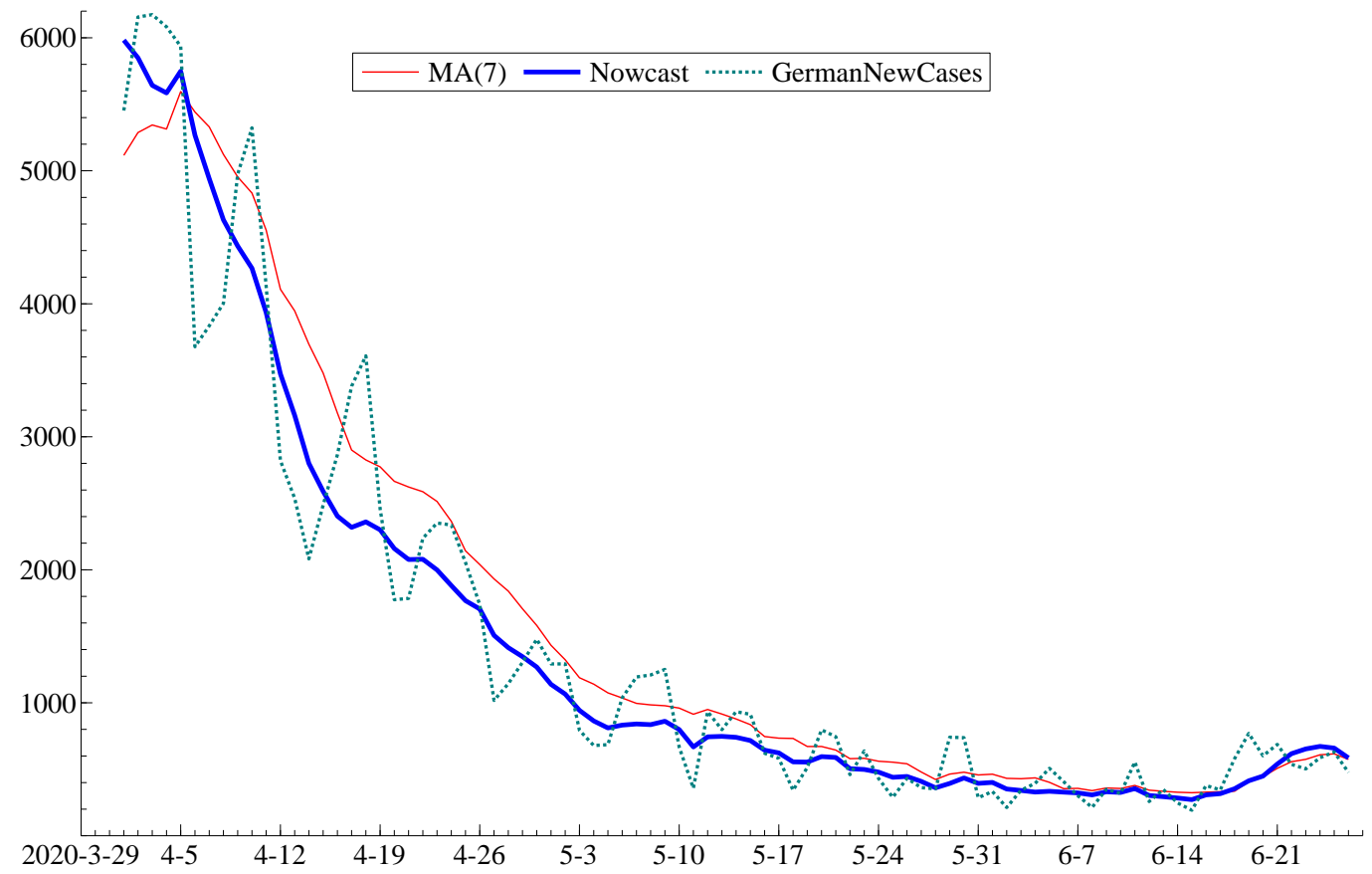

Fig. 1. German new cases from 29th March till 26th June (data sourced from ECDC) showing nowcasts from model and 7-day moving averages.

to gradually discount past observations. Hence it can respond more quickly. Figure 1 shows nowcasts of the underlying trend in new cases produced by the model for Germany (ECDC data), together with the MA7. The attraction of the model is clear and, of course, it also has the advantage of being able to produce forecasts. Lagging the MA7 so it is centred at $t-3$ would shift it more in line with the observations but at the cost of losing the last three observations.

\subsection{Forecasting the trend in future observations}

The forecasts of the trend in future values of $\ln g_{t}$ in the dynamic Gompertz model are given by $\delta_{T+\ell \mid T}=\delta_{T \mid T}+\gamma_{T \mid T} \ell, \ell=1,2, .$. , where $\delta_{T \mid T}$ and $\gamma_{T \mid T}$ are the KF estimates of $\delta_{T}$ and $\gamma_{T}$ at the end of the sample. Forecasts of the trend in the daily observations, $y_{t}$, may be obtained from a recursion for the trend in their cumulative total, $Y_{t}$, namely

$$
\mu_{T+j \mid T}=\mu_{T+j-1 \mid T}\left(1+g_{T+j \mid T}\right)=\mu_{T+j-1 \mid T}\left(1+\exp \delta_{T+j \mid T}\right), \quad j=1,2, . ., \ell,
$$


with $\mu_{T \mid T}=Y_{T}$. The trend in the daily figures is then

$$
\mu_{y, T+\ell \mid T}=g_{T+\ell \mid T} \mu_{T+\ell-1 \mid T} \quad, \quad \ell=1,2, . .
$$

Combining the above equations gives

$$
\begin{aligned}
& \mu_{y, T+\ell \mid T}=Y_{T} \exp \delta_{T+\ell \mid T} \prod_{j=1}^{\ell-1}\left(1+\exp \delta_{T+j \mid T}\right), \quad \ell=2,3, . . \\
& \mu_{y, T+1 \mid T}=Y_{T} \exp \delta_{T+1 \mid T} .
\end{aligned}
$$

Daily effects can be added to $\delta_{t}$. In this case forecasts of the observations themselves, that is $\widehat{y}_{T+\ell \mid T}$ and $\widehat{Y}_{T+\ell \mid T}$, are given by adding the filtered value of the daily component to the trend component, $\delta_{T+\ell \mid T}$.

The conditional distribution of future values of the trend, $\delta_{T+\ell}$, in $\ln g_{T+\ell}$ is Gaussian. The conditional distribution of $\exp \delta_{T+\ell}$ is therefore lognormal but, for more than one-step ahead, the distribution of the corresponding trend in the observations is not lognormal because of the presence of the unknown cumulative total in our equation for the underlying trend which is $\mu_{y, T+\ell}=g_{T+\ell} Y_{T+\ell-1}, \ell=2,3$. However since $Y_{t}$ changes relatively slowly it may be possible to ignore its effect by treating it as fixed.

An alternative to working with the distribution of the trend of the observations, is to convert a prediction interval for $\ln g_{T+\ell}$ into one for $\mu_{y, T+\ell}$ by replacing $\delta_{T+j \mid T}$ in (9) by $\delta_{T+j \mid T} \pm z \cdot \sigma_{\delta, T+j \mid T}$, where $\sigma_{\delta, T+j \mid T}^{2}$ is the conditional variance of $\delta_{T+j}$ and $z$ is a constant such as one or two. Again, with $Y_{t}$ changing slowly, there may be a case for simply constructing a prediction interval from (10) by replacing $\delta_{T+\ell \mid T}$ by $\delta_{T+\ell \mid T} \pm z \cdot \sigma_{\delta, T+j \mid T}$ for $\ell=1,2,3, \ldots$ If a prediction interval for the observations themselves is wanted, the standard deviation $\sigma_{\delta, T+j \mid T}$

may be replaced by $\sqrt{\sigma_{\delta, T+j \mid T}^{2}+\sigma_{\varepsilon}^{2}}$ in the preceding formulae. Allowance may also need to be made for a daily component.

\subsection{Nowcasts of the trend in daily observations}

The nowcast for the trend in $y_{t}$ is

$$
\mu_{y, t \mid t}=Y_{t-1} \exp \delta_{t \mid t}, \quad t=t^{\prime}, \ldots, T
$$

Using the current rather than the lagged cumulative total, that is $Y_{t} \exp \delta_{t \mid t}$, makes virtually no difference once $Y_{t}$ has become relatively large. Since $\delta_{t}$ is conditionally Gaussian, $\exp \delta_{t}$ is lognormal and a credible interval may be produced if required. 


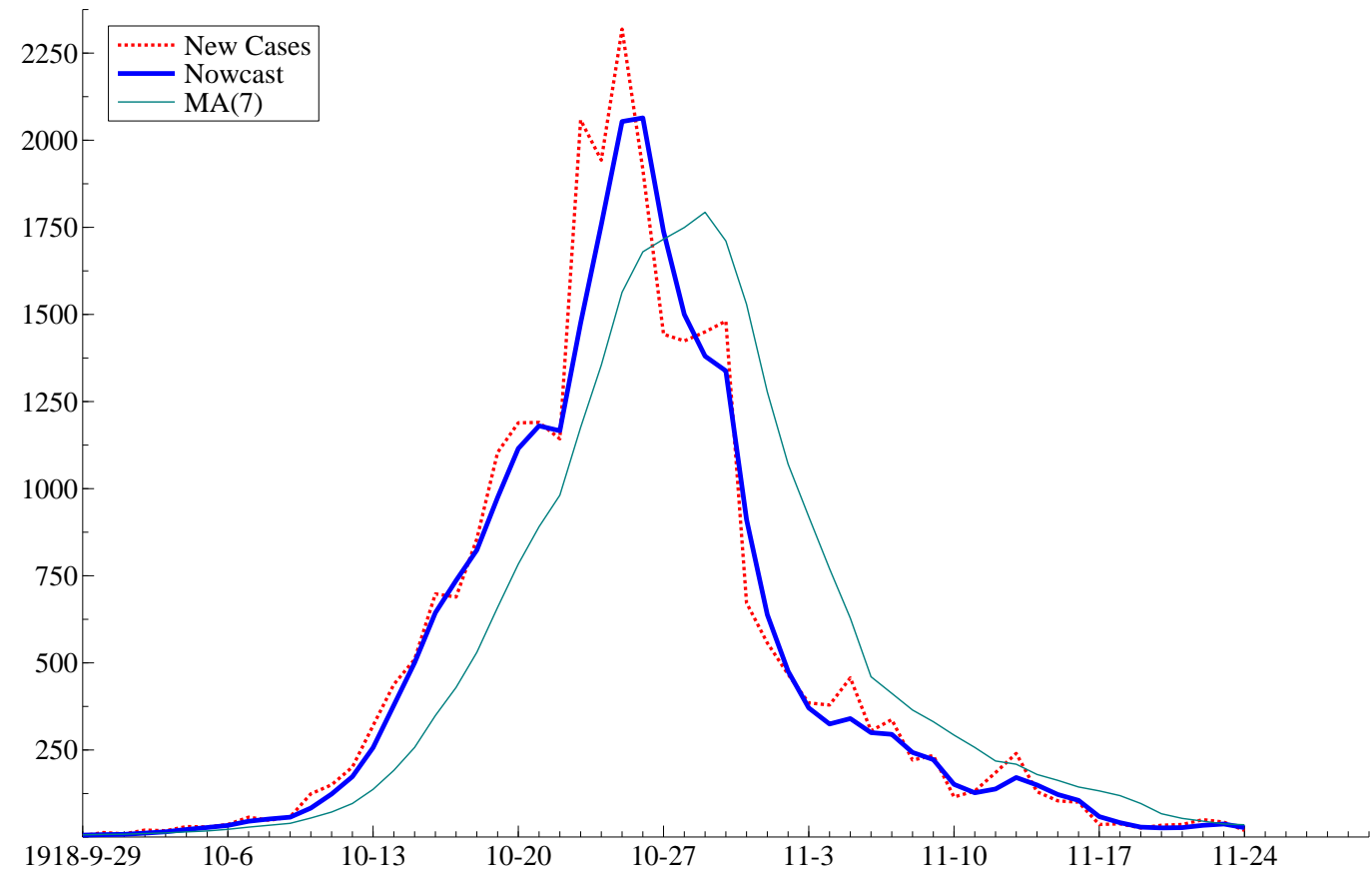

Fig. 2. Nowcasts and 7-day moving averages for San Francisco flu from 29th September till 24th October 1918.

Example Daily cases of influenza $a^{4}$ in San Francisco during the worldwide outbreak of Spanish flu in 1918 show exponential growth in the upward phase; see Chowell et al (2007). Consequently a plot of the logarithm of the growth rate (LDL) shows very little downward movement at first. Fitting the Gaussian dynamic Gompertz to the whole series gives $q=0.05$. The slope in LDL adapts so it is close to zero in early October and then falls so as to capture the downward phase. Figure 2 contrasts the nowcasts with a seven day moving average, which lags behind the observations throughout.

\section{COVID-19 in the UK and regions}

Our empirical focus is on trends in new cases in the UK, its nations and English regions. We focus on early 2021, when the new strain of COVID-19 was the leading cause of increase in infection rates, initially in the south-east of England.

The daily counts of COVID-19 cases are based on the results of laboratory-based or swab tests for the presence of SARS-CoV-2 virus in specimens taken from people, as well

\footnotetext{
${ }^{4}$ The data are supplementary material to the article by Chowell (2007) https://www.ncbi.nlm.nih. gov/pmc/articles/PMC2358966/
} 
as results of antibody serology tests. The new cases data are available by the date the specimen was collected (the specimen date series) and by the date the testing process was completed and the case was first included in the published totals (published date series). A key issue is how information in the specimen date series can be combined with that in the published date series. There are pronounced daily effects in the specimen date series, which however can be accommodated in a model with seasonal effects. More importantly the specimen date series is subject to substantial reporting delays and revisions. Figure 3 illustrates the extent of revisions over time of the specimen date series, with reference to the same data as revised three weeks in the future by when revisions are almost complete and very small. To be precise, let $y_{t}^{(v)}$ denote the $v$ day ahead update (or vintage) for $y_{t}$, the specimen cases recorded on date $t$. Thus, the current vintage of the data is given by the series: $y_{t}^{(t)}, y_{t-1}^{(t)}, \ldots, y_{1}^{(t)}$. The date $r$ revision to the current vintage of specimen cases for date $i$ is defined as: $r e v_{i}^{(r)}=y_{i}^{(r)}-y_{i}^{(t)}$. Figure 3 presents the revisions three weeks ahead for the last four entries of the current vintage, $r e v_{t}^{(t+21)}, \ldots, r e v_{t-3}^{(t+21)}$. It is evident that except in the neighbourhood of Christmas day and New Year's day when data quality was very poor, revisions were substantially complete within three days.

A technical issue led to a large number of infections that occurred between 25th September and 2nd October 2020 going unrecorded and then being assigned to 3rd and 4th October, thereby creating an artificial spike. Rather than attempting to reallocate observations we start our analysis with data published on 5th October 2020. Cases by specimen date were not affected by the above issue. On 27th November 2020 another technical issue led to the total number of people who tested positive being revised down.

We fit models to the logarithm of the growth rate of new cases as measured by the specimen date up to and including time $t-3$ and report nowcasts and forecasts of $g_{y, t+h}$ and $\widetilde{R}_{t+h}^{e}$ for $h=-3,0,7$ and 14 . For models fitted to new cases measured by published date, we report nowcasts and forecasts for $h=0,7$ and 14 . Note that the published data for time $t$ is actually released at $t+1$.

The forecasts we generate make no assumptions about the effects of measures imposed to control the spread of the epidemic. Thus the forecasts made at the start of the year overshoot the eventual numbers. As the restrictive measures begin to bite, the forecasts made by the model adapt.

\subsection{Nowcasts and forecasts in January 2021}

Tables 1 and 2 present, at weekly intervals starting on 28th December 2020, nowcasts and forecasts of the growth rate of new cases, $g_{y, t}, g_{y, t+7}$ and $g_{y, t+14}$, and of the reproduction 


$$
-\operatorname{rev}_{t}^{(\mathrm{t}+21)}-\operatorname{rev}_{\mathrm{t}-1}^{(\mathrm{t} 21)}-\operatorname{rev}_{\mathrm{t}-2}^{(\mathrm{t}+21)} \cdots \operatorname{rev}_{\mathrm{t}-3}^{(\mathrm{t} 21)}
$$

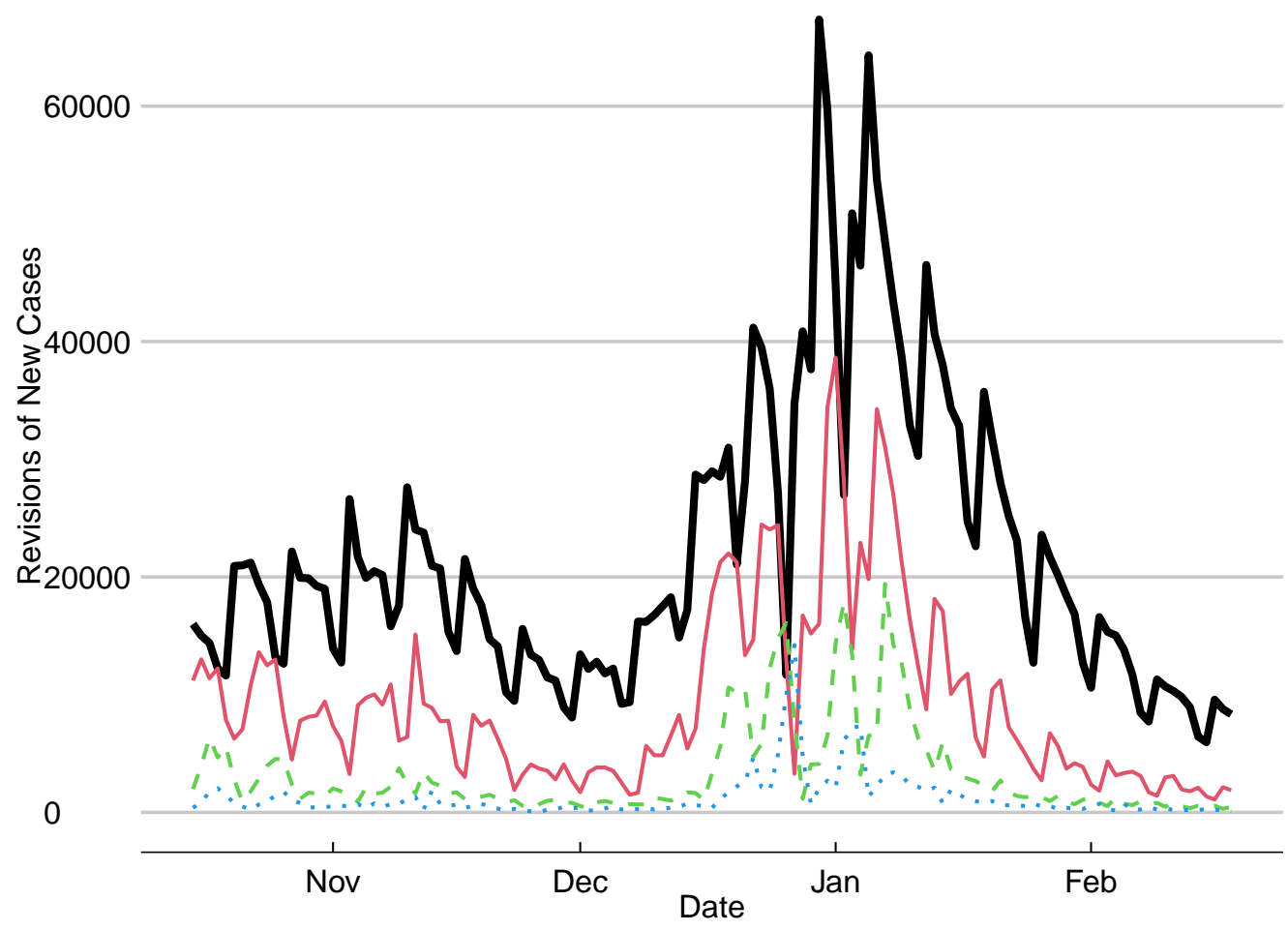

Fig. 3. Differences between new cases in the specimen date series at $t$, and the same data revised three weeks in the future.

number, $R_{t}, R_{t+7}$ and $R_{t+14}$, for England. Table 1 uses the publication date series while Table 2 is for the specimen date series. The projections from both series are based on trends without daily effects, and show broadly similar patterns of accelerating growth rates before Christmas that increased through the New Year to the first observations in 2021. The lock down of 5th January 2020 brought both sets of growth rates and reproduction numbers down to the same broad range within a week. The growth rates estimated from both the publication date series and the specimen date series have continued to be negative since then.

Figure 4 gives the forecasts of new cases based on publication date series for England, including the daily effect. Figure 5 gives the forecasts based on the specimen date series. Vertical dashed lines denote the end of the estimation sample. These figures demonstrate that once past the imposition of the January lockdown, the model adapted quickly to the change in the series and in a relatively stable environment provided accurate forecasts. 
Table 1: England: $g_{y, t+h}$ and $\widetilde{R}_{t+h}^{e}$ based on publication date series

\begin{tabular}{|c|c|c|c|}
\hline \multirow[t]{2}{*}{$\mathrm{t}$} & \multicolumn{3}{|c|}{$\mathrm{h}$} \\
\hline & 0 & 7 & 14 \\
\hline \multicolumn{4}{|l|}{$g_{y}$} \\
\hline $2020-12-28$ & 5.25 & 5.76 & 6.40 \\
\hline 2021-01-04 & 5.47 & 6.04 & 6.76 \\
\hline 2021-01-11 & -1.31 & -1.66 & -1.95 \\
\hline 2021-01-18 & -4.78 & -5.17 & -5.43 \\
\hline 2021-01-25 & -7.02 & -7.31 & -7.48 \\
\hline 2021-02-01 & -4.99 & -5.16 & -5.27 \\
\hline \multicolumn{4}{|l|}{$\underline{R}$} \\
\hline $2020-12-28$ & 1.23 & 1.26 & 1.29 \\
\hline 2021-01-04 & 1.24 & 1.27 & 1.31 \\
\hline 2021-01-11 & 0.95 & 0.94 & 0.92 \\
\hline 2021-01-18 & 0.83 & 0.81 & 0.80 \\
\hline 2021-01-25 & 0.76 & 0.75 & 0.74 \\
\hline 2021-02-01 & 0.82 & 0.81 & 0.81 \\
\hline
\end{tabular}

Table 2: England: $g_{y, t+h}$ and $\widetilde{R}_{t+h}^{e}$ based on specimen date series.

\begin{tabular}{lrrrrr}
\hline \multicolumn{1}{c}{$\mathrm{t}$} & \multicolumn{5}{c}{$\mathrm{h}$} \\
\cline { 1 - 3 } \cline { 5 - 6 }$\frac{g_{y}}{2020-12-28}$ & & 2.02 & 2.03 & 2.06 & 2.10 \\
$2021-01-04$ & & 4.24 & 4.37 & 4.73 & 5.13 \\
$2021-01-11$ & & -2.39 & -2.60 & -3.01 & -3.31 \\
$2021-01-18$ & & -3.96 & -4.14 & -4.46 & -4.68 \\
$2021-01-25$ & -4.35 & -4.47 & -4.70 & -4.85 \\
$2021-02-01$ & -4.62 & -4.70 & -4.85 & -4.95 \\
& & & & \\
\hline & & & & \\
$\underline{R}$ & & & & \\
$2020-12-28$ & 1.08 & 1.08 & 1.09 & 1.09 \\
$2021-01-04$ & 1.18 & 1.19 & 1.21 & 1.23 \\
$2021-01-11$ & 0.91 & 0.90 & 0.89 & 0.88 \\
$2021-01-18$ & 0.85 & 0.85 & 0.84 & 0.83 \\
$2021-01-25$ & 0.84 & 0.84 & 0.83 & 0.82 \\
$2021-02-01$ & 0.83 & 0.83 & 0.82 & 0.82 \\
\hline
\end{tabular}



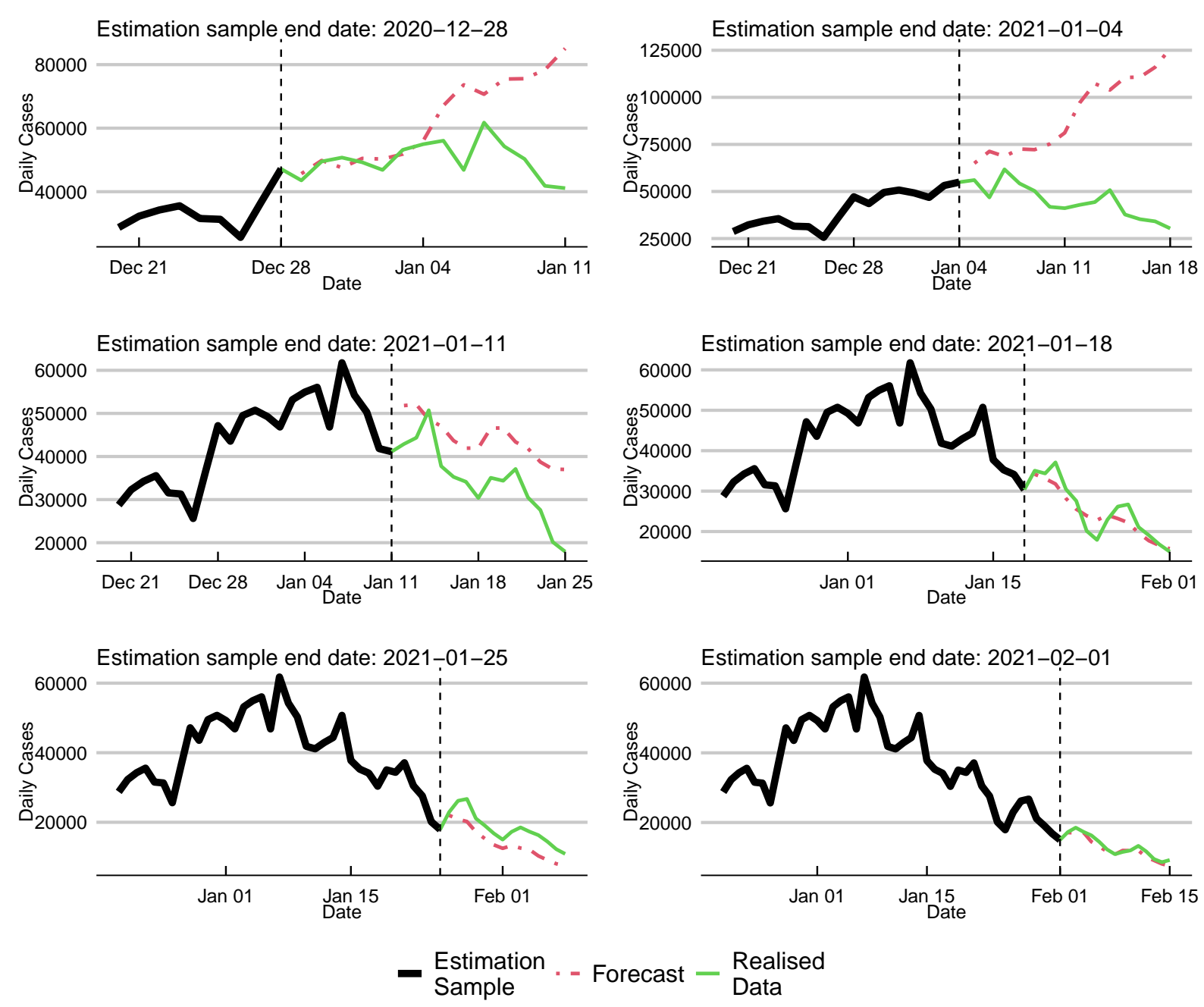

Fig. 4. England: forecasts of new cases based on publication date series.

\subsection{Forecast accuracy}

We assess forecast accuracy using Mean Absolute Percentage Error (MAPE) over the 14day period from the date on which the data is released. For the publication date series we evaluate forecasts against subsequent realisations of the same series, whereas for specimen date series we evaluate forecasts against the first vintage with a release date that allows the first major revisions to vanish from the evaluation sample. This also maintains a fixed number of days for each evaluation date relative to the forecast origin for revisions to enter the evaluation sample. Thus, evaluation data for the specimen data series with vintage dated $t$ requires evaluation data of vintage $(v)$ from $y_{t-2}^{(v)}$ to $y_{t+14}^{(v)}$, because we truncate the estimation sample at $y_{t-3}^{(t)}$. We choose a vintage of $v=t+17$ to allow for the discarding of the heavily revised data at $t+15$ to $t+17$. Where this is not possible due to lack of data, we 

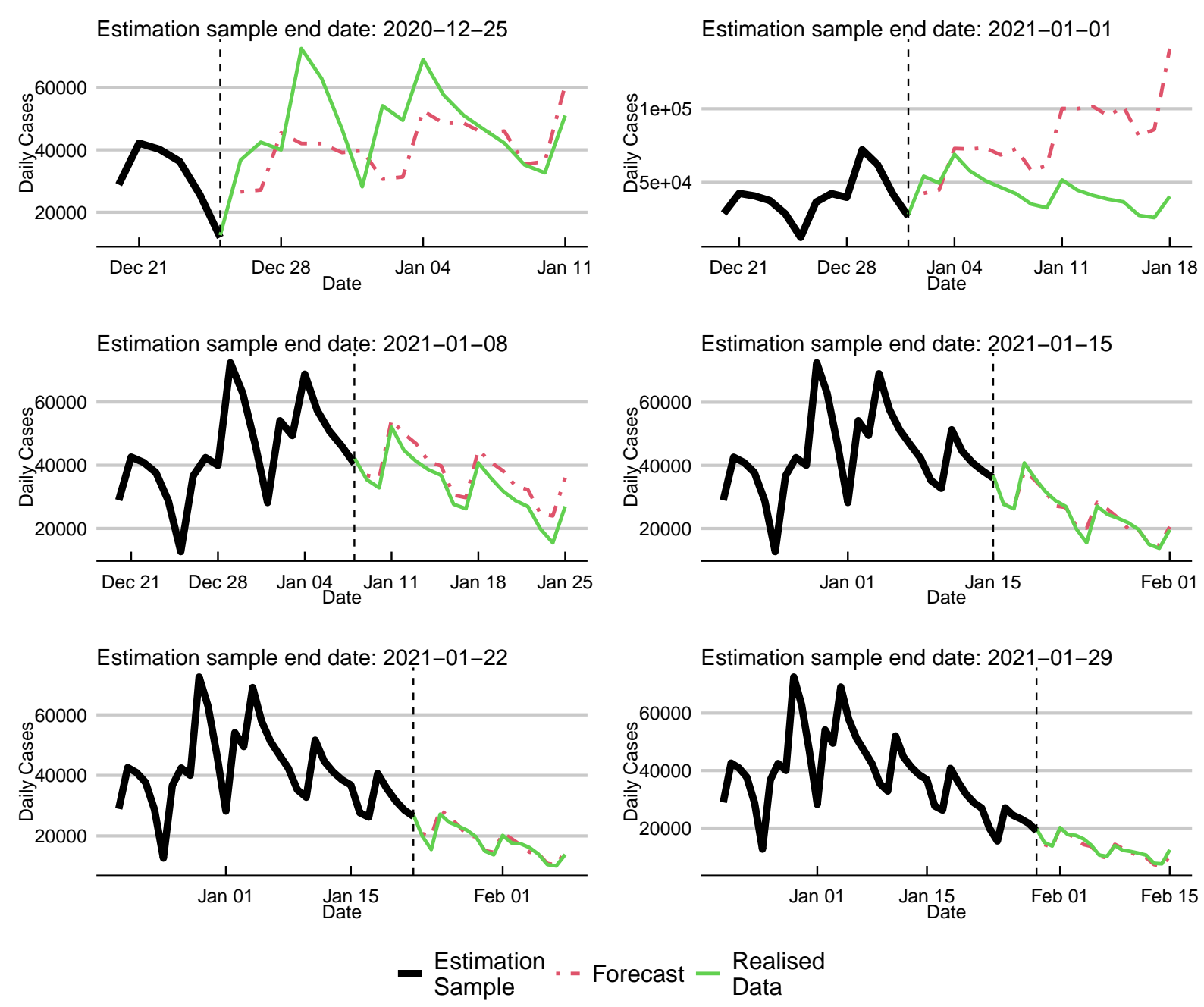

Fig. 5. England: forecasts of new cases based on specimen date series.

set $v$ equal to the last date upon which data is available (which in Figure 5 is 23rd February 2021). For publication date series ending at time $t$, we require evaluation data from $y_{t+1}^{(j)}$ until $y_{t+14}^{(j)}$. As the publication data is just the first release of the specimen data this results in the evaluation sample $y_{t+1}^{(t+1)}, \ldots, y_{t+14}^{(t+14)}$.

Table 3 reports the MAPE for the forecasts of new cases based on the publication date series. Recall that data on new cases at $t$ becomes available at $t+1$ in the publication date series. Table 4 reports the corresponding forecasts for the specimen date series. Accuracy is comparable for nowcasts and forecasts generated from the two series. Once the shocks to data quality over Christmas and the New Year are past and the initial effect of the January lockdown has worked through, both the 7 and 14 day ahead forecasts become more accurate. 
Table 3: England: Accuracy (MAPE) of forecasts of publication date series of new cases.

\begin{tabular}{|c|c|c|c|}
\hline \multirow[t]{2}{*}{$\mathrm{t}$} & \multicolumn{3}{|c|}{$\mathrm{h}$} \\
\hline & 1 & 7 & 14 \\
\hline $2020-12-28$ & 4.8 & 3.7 & 28.6 \\
\hline 2021-01-04 & 15.9 & 47.7 & 119.1 \\
\hline 2021-01-11 & 20.8 & 21.7 & 36.1 \\
\hline 2021-01-18 & 2.5 & 11.7 & 9.7 \\
\hline $2021-01-25$ & 3.9 & 17.5 & 24.8 \\
\hline 2021-02-01 & 1.0 & 5.1 & 6.5 \\
\hline
\end{tabular}

Table 4: England: Accuracy (MAPE) of forecasts of specimen date series of new cases.

\begin{tabular}{|c|c|c|c|c|}
\hline \multirow[t]{2}{*}{$\mathrm{t}$} & \multicolumn{4}{|c|}{ h } \\
\hline & -2 & 0 & 7 & 14 \\
\hline $2020-12-28$ & 27.7 & 25.8 & 31.4 & 22.2 \\
\hline 2021-01-04 & 21.3 & 12.2 & 47.0 & 102.1 \\
\hline 2021-01-11 & 3.9 & 5.9 & 9.2 & 16.1 \\
\hline 2021-01-18 & 0.5 & 3.4 & 5.7 & 5.1 \\
\hline 2021-01-25 & 4.2 & 13.1 & 6.6 & 6.0 \\
\hline 2021-02-01 & 5.0 & 2.8 & 4.3 & 6.0 \\
\hline
\end{tabular}

\subsection{Comparison with $R$ published by DHSS and SAGE}

The benchmarks for our results are the estimates of the growth rate and $R$ values published jointly by the Department of Health and Social Care and the Scientific Advisory Group for Emergencies, based on contributions by different modelling groups using a variety of data sources. Estimates can vary between different models and are presented as ranges. For example, on 5 February 2021 the published range estimates for England were [0.7, 0.9] for $R_{t}$ and $[-5 \%,-2 \%]$ for the growth rate. Note that due to time delays, estimates reflect transmission of the disease over the past few weeks.

Figure 6 presents the model based estimates of $R$ for England using the publication and specimen date series, and for comparison, the empirical estimate of $R$ based on the RKI estimator (Section 2.3), as well as the range estimates of $R$ published by SAGE, obtained from https://www.gov.uk/guidance/the-r-number-in-the-uk. The model based estimates of $R$ are quicker to reveal the effect of the January lockdown on infection transmission than the SAGE estimates.

Analysis and results corresponding to the above for the UK, other nations and the regions of England are presented in an online supplement to this paper. 


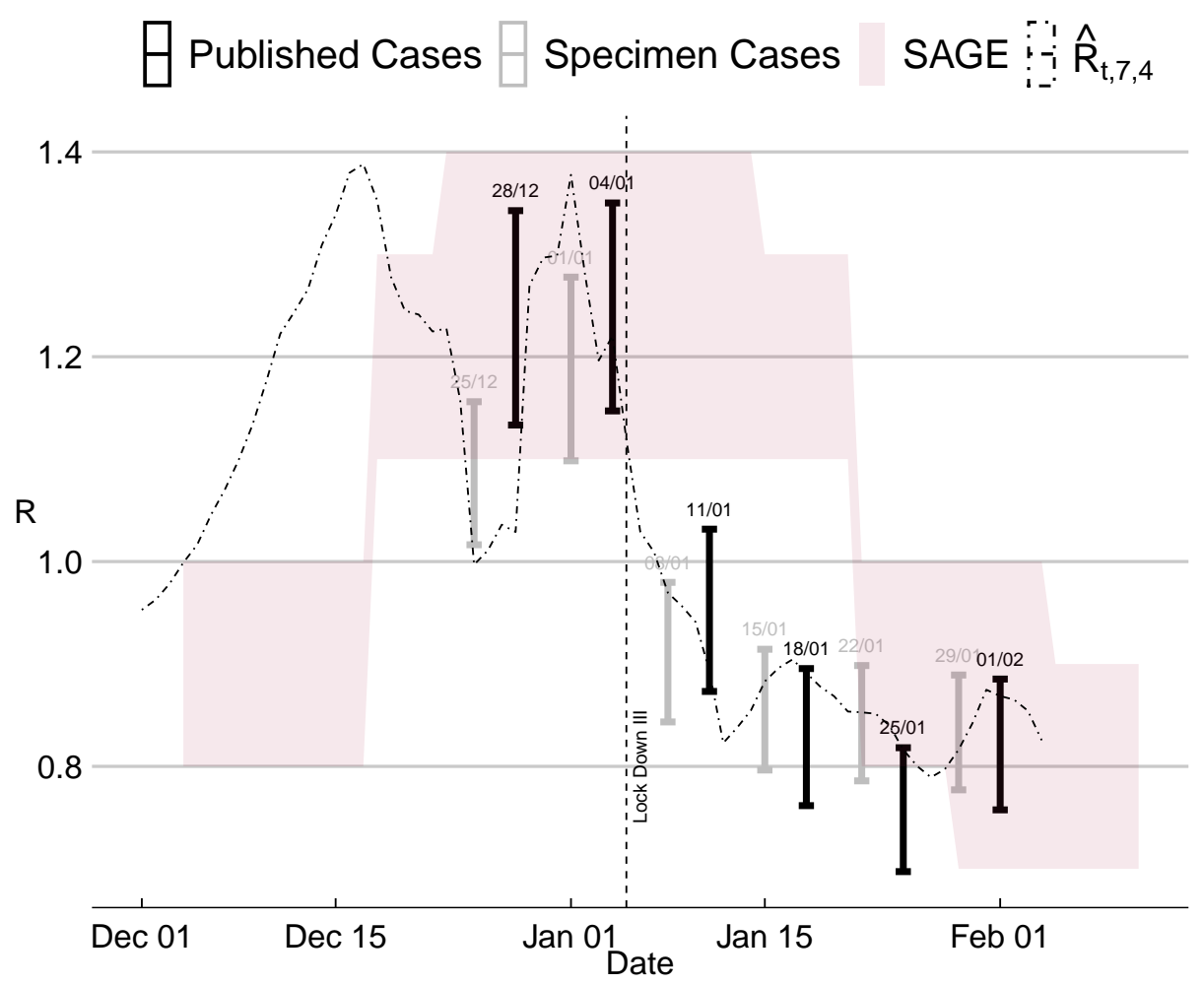

Fig. 6. Estimates of $\mathrm{R}$ based on publication and specimen date series vis-a-vis $\mathrm{R}$ ranges published by SAGE.

\section{Combining observations by publication date and spec- imen date}

Methods of dealing with preliminary observations and observations at different vintages have long been employed in econometrics. Our treatment of published and specimen data owes much to this literature but there are some novel features, primarily concerned with time-varying slopes and the notion of balanced growth. The techniques may be generalized to deal with situations where growth may not be balanced. Similar techniques may be employed when one series is a leading indicator of the other.

The bivariate model has observations on the first variable (published series), which is effectively a leading indicator, available at time $t$, whereas the second (specimen series) is only observed after $k$ periods. Thus at time $t$ the observations on $\ln g_{2 t}$ are missing for $t-k+1, \ldots, t$. The model is 


$$
\begin{array}{rlrl}
\ln g_{1 t} & =\delta_{t}+\psi_{1 t}+\varepsilon_{1 t}, & t=1, \ldots, T, \\
\ln g_{2 t} & =\delta_{t}+\bar{\delta}+\psi_{2 t}+\varepsilon_{2 t} & & \\
\psi_{j t} & =\phi_{j} \psi_{j, t-1}+\eta_{j t}, & & \eta_{j t} \sim N I D\left(0, \sigma_{\eta j}^{2}\right) .
\end{array}
$$

where $\bar{\delta}$ is a constant term; $\delta_{t}$ will contain a constant that can be (arbitrarily) assigned to series one. As in the univariate models of the last section, the trend, $\delta_{t}$, is an IRW that contains the information needed to estimate the underlying movements in the growth rate of the target series, $g_{2, y, t}$. All disturbances, including $\varepsilon_{1 t}$ and $\varepsilon_{2 t}$, are Gaussian and assumed to be mutually as well as serially independent. Provided $\left|\phi_{j}\right|<1, j=1,2$, the series are cointegrated of order $(2,2)$, that is $C I(2,2)$, with balanced growth. The difference $\ln g_{1 t}-\ln g_{2 t}$ is a stationary $\operatorname{ARMA}(2,2)$ process, but setting $\phi_{1}=\phi_{2}$ gives an $\operatorname{AR}(1)$ plus noise.

The Kalman filter provides the (filtered) state estimates needed to compute the nowcasts for $g_{2, y, T}, R_{T}$ and $y_{2 T}$. As new observations become available these nowcasts are updated by the KF. Smoothed estimates of variables from $t=T-k+1$ to $t=T-1$ can be computed if needed. Forecasts of the state beyond time $T$ are made by the predictive KF and corresponding forecasts of $R_{t}$ and $y_{2 t}$ can be formed. Daily effects are included in the applications and are handled in (11) by adding a 'seasonal' component.

A modified version of the model confines the $\mathrm{AR}(1)$ component to the first variable so that

$$
\begin{array}{rlrl}
\ln g_{1 t} & =\delta_{t}+\psi_{t}+\varepsilon_{1 t}, & t & t, \ldots, T, \\
\ln g_{2 t} & =\delta_{t}+\bar{\delta}+\varepsilon_{2 t} & & \\
\psi_{t} & =\phi \psi_{t-1}+\eta_{1 t}, & \eta_{t} \sim \operatorname{NID}\left(0, \sigma_{\eta}^{2}\right) .
\end{array}
$$

An advantage of this simplication is that the signal-noise ratio in the target can be compared with that of a univariate model and, if desired, set to a pre-assigned value.

Nowcasts of the trend in observations are obtained from recursions similar to those in sub-section 2.4, except that filtered estimates of $\delta_{t}$ are replaced by smoothed ones. Thus

$$
\mu_{T-k+\ell \mid T}=\mu_{T-k+\ell-1 \mid T}\left(1+g_{T-k+\ell \mid T}\right)=\mu_{T+\ell-1 \mid T}\left(1+\exp \delta_{T-k+\ell \mid T}\right), \quad \ell=1,2, . ., k
$$

with $\mu_{T \mid T-k}=Y_{T}$, so

$$
\mu_{y, T+\ell \mid T}=g_{T+\ell \mid T} \mu_{T+\ell-1 \mid T}, \quad \ell=1,2, . ., k
$$




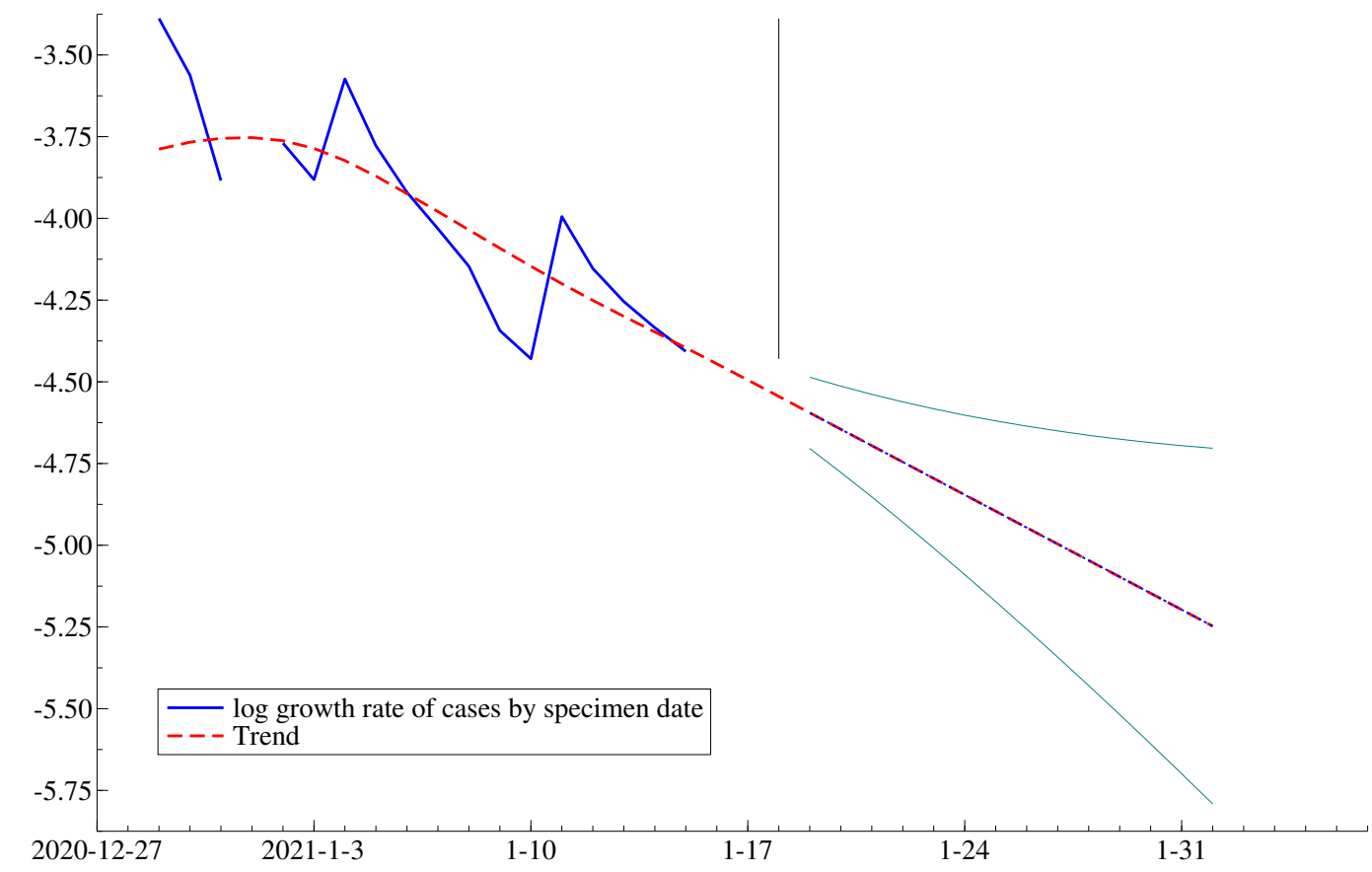

Fig. 7. Forecasts of trend in specimen cases made with observations up to 15 th January 2021 but using published data up to 18th January 2021.

The recursions can be continued to give forecasts. The difference is that when $\ell>k$, the $\delta_{T-k+\ell \mid T}^{\prime} s$ are forecasts, not smoothed estimates.

The bivariate model, (12), was fitted to data available on 19th January. The observations start on October 4th and finish on January 18th for the published series and on January 15th for specimen series. The Christmas day and New Year specimen observations were treated as missing. The reasons for omitting the last three specimen dated figures were set out in sub-section 3. Estimation details can be found in Appendix B.

The estimates of $g_{y, T}$ and $R_{T}^{e}$ for the specimen dated series are $-0.040(0.026)$ and 0.85 (0.10) respectively. These are the same (to two decimal places) as the estimates for the univariate series. Figure 7 shows nowcasts, from 16th to 18th January, and forecasts, from 19th, of the trend in specimen data. The prediction interval for the observations on $\ln g_{t}$ is one standard deviation either side of its predicted trend. (A daily effect was not included in the model.) 


\section{Conclusions and future directions}

This article has demonstrated the way in which our new time series models are able to track the progress of the COVID-19 epidemic in the UK in early 2021. The models are not only simple and transparent, but are able to adapt quickly to changes in key series. This ability to respond in a timely fashion is illustrated by the comparison of our estimates of the current $\mathrm{R}$ number with those produced by SAGE. The complexity of the behavioural response to lockdown and the restrictive measures imposed by the Tier system in different areas in late 2020 makes a formal structural modelling difficult. The roll out of the vaccine adds yet more complexity. Our models track these changes and project forward to make short-term forecasts of the situation over the next few weeks. ${ }^{5}$ Models are estimated for the four UK nations and for the regions within England. All the models have the same form.

We show how multivariate generalizations of our models can combine information in different series, some of which are subject to substantial revisions. The approach derives from econometric approaches to handling different vintages, but there are some novel technical features. The methods are new to epidemiology. We demonstrate that joint modelling of

published and specimen dated observations on new cases can be accomplished without too much difficulty. The methods may be adapted to use some time series as leading indicators for others. Further work on using new cases as a leading indicator of admissions and deaths is currently underway.

\section{Acknowledgements}

Comments and suggestions from Leopoldo Catania, Jagjit Chadha, Radu Cristea, Daniela de Angelis, Michael Höhle, Jonas Knecht, Rutger-Jan Lange, Franco Peracchi, Jerome Simons and a referee are gratefully acknowledged; of course they bear no responsibility for opinions expressed or mistakes made. Some of the ideas were presented at the NIESR conference in November 2020 and at University of Cambridge BSU seminar in January 2021.

$\mathrm{AH}$ is grateful to the University of Cambridge Keynes Fund for support on the project Persistence and Forecasting in Climate and Environmental Econometrics. 


\section{References}

Anesti, N., Galvão, A. B., Miranda-Agrippino, S., 2021. Uncertain kingdom : nowcasting gdp and its revisions. Journal of Applied Econometrics, to appear .

Avery, C., Bossert, W., Clark, A., Ellison, G., Ellison, S., 2020. An economist's guide to epidemiology models of infectious disease. Journal of Economic Perspectives, 34(4), 79104 .

Chowell, G., Nishiura, H., Bettencourt, L. M. A., 2007. Comparative estimation of the reproduction number for pandemic influenza from daily case notification data. The Journal of the Royal Society Interfaces, 4(12), 155-166 .

Durbin, J., Koopman, S., 2012. Time Series Analysis by State Space Methods. Oxford: Oxford University Press.

Harvey, A., 1989. Forecasting, Structural Time Series Models and the Kalman Filter. Cambridge: Cambridge University Press.

Harvey, A., 2020. Time series models for epidemics: leading indicators, control groups and policy assessment. NIESR Discussion Paper, Number: 517 .

Harvey, A., Chung, C., 2000. Estimating the underlying change in unemployment in the uk, with discussion. Journal of the Royal Statistical Society Series A, 163, 303-39 .

Harvey, A., Kattuman, P., 2020a. Time series models based on growth curves with applications to forecasting coronavirus. Harvard Data Science Review, Special issue 1 - COVID-19. https://hdsr.mitpress.mit.edu/pub/ozgjx0yn.

Harvey, A., Kattuman, P., 2020b. A farewell to R: Time series models for tracking and forecasting epidemics. CEPR working paper, Issue 51, 7th October. https://cepr.org/ content/covid-economics.

Höhle, M., an der Heiden, M., 2014. Bayesian nowcasting during the stec o104:h4 outbreak in germany. Biometrics, 70, 993-1002 .

Ioannidis, J., Cripps, S., Tanner, M., 2020. Forecasting for covid-19 has failed. International Journal of Forecasting. https://doi.org/10.1016/j.ijforecast.2020.08.004.

Koopman, S., Lit, R., Harvey, A., 2020. STAMP 8.4: Structural time series analyser, modeller and predictor. Timberlake Consultants. 
Wallinga, J., Lipsitch, M., 2000. How generation intervals shape the relationship between growth rates and reproductive numbers. Proceedings of the Royal Society B, 274, 599-604

\section{A: Data sources}

The data for the UK was obtained from Public Health England's (PHE) Coronavirus toolkit (https://coronavirus.data.gov.uk/developers-guide). Archived, or, real-time data is currently not available in the first release, 'v1', of their Application Programming Interface (API). Discussion of access to archived data is summarised in this github ticket: https://github.com/publichealthengland/coronavirus-dashboard/issues/241. Towards the later part of 2020 PHE released an experimental version, 'v2', of their API which has archived data from 12th August 2020 onwards. All archived data is taken from this endpoint: https://api.coronavirus.data.gov.uk/v2/. It is worth restating their disclaimer that this is an experimental endpoint and "subject to active development and may become unstable or unresponsive without prior notice".

\section{B: Estimation for bivariate model for publication and specimen data series}

The prediction error variances for specimen and published were 0.0134 and 0.0172 respectively with a correlation of 0.077 . The slope variances were constrained to be the same and $q$ was set at 0.015 for the specimen series. The estimated AR coefficient in the published series was 0.672 and its variance was 0.0056 . The irregular variances for specimen and published were 0.0082 and 0.0091 respectively with a correlation of -0.190 .

Figure 8 shows the residual ACFs and histograms. The diagnostic statistics were ${ }^{6}$ as follows for specimen : $r(1)=0.20, Q(18)=25.41, B S=9.87$ and $H=3.95$ and for published: $r(1)=-0.07, Q(18)=20.21, B S=0.38$ and $H=0.90$. There is some remaining serial correlation, but not a great deal. Fitting an AR(1) to the specimen series as well as to the published series may reduce $r(1)$. The greater stability in the published series is reflected in the smaller BS normality test statistic.

\footnotetext{
${ }^{6} \mathrm{r}(1)$ is the autocorrelation at lag one, $\mathrm{Q}(\mathrm{P})$ is Box-Ljung statistic with $\mathrm{P}$ autocorrelations, BS is the Bowman-Shenton normality statistic and $\mathrm{H}$ is a heteroscedasticity statistic constructed as the ratio of the sum of squares in the last third of the sample to the sum of squares in the first third.
} 

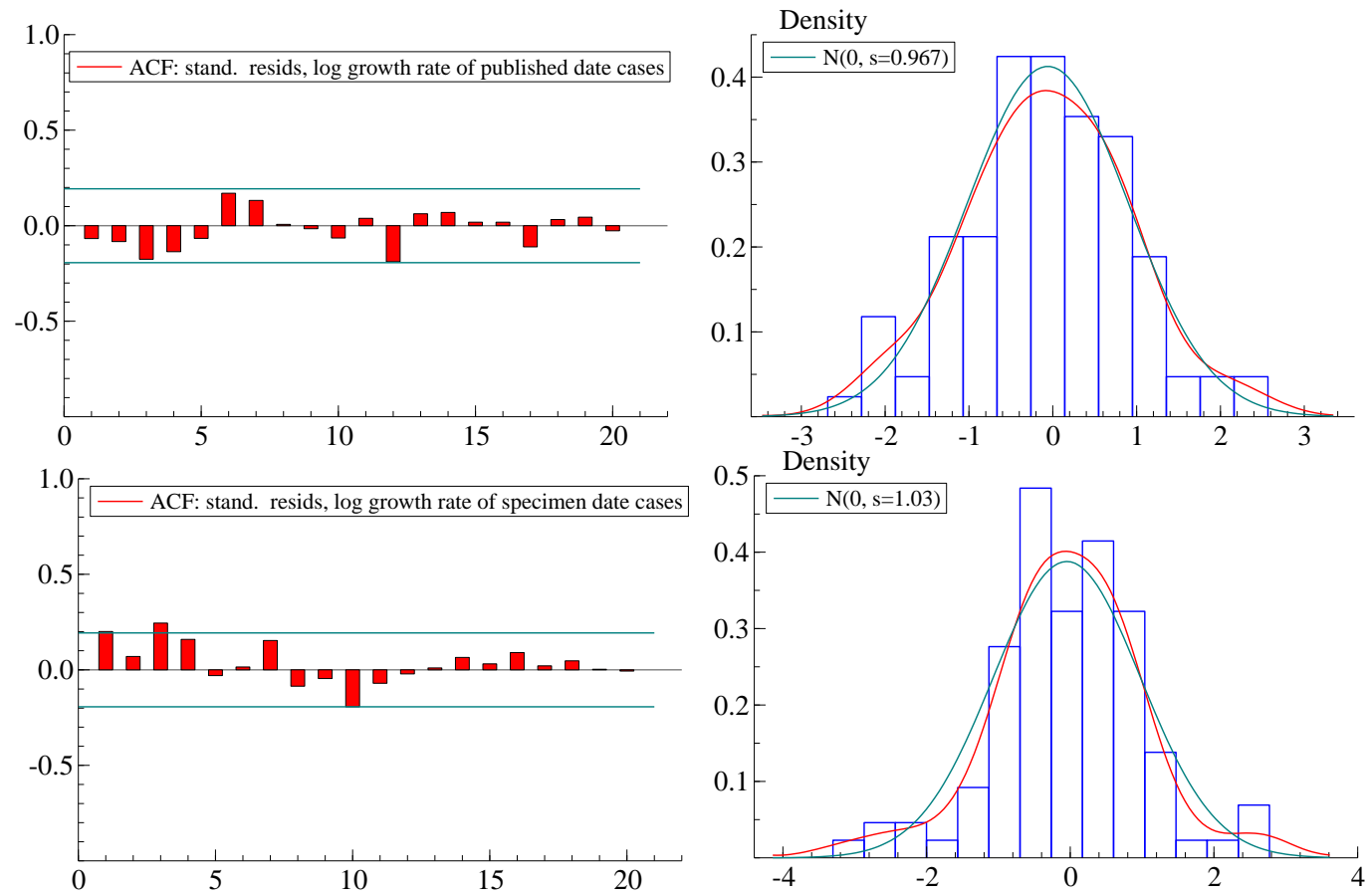

Fig. 8. Residuals from bivariate model fitted to data up to 19th January 2021.

The output for the state vector shows that the slopes on January 18th are almost identical for the two series; for specimen data -0.0506 (0.0260) and for published it is -0.0502 (0.0261). The difference arises because, although STAMP is able to constrain the variances of the slopes to be the same, it is currently unable to set the deterministic parts of the slope to be equal. 